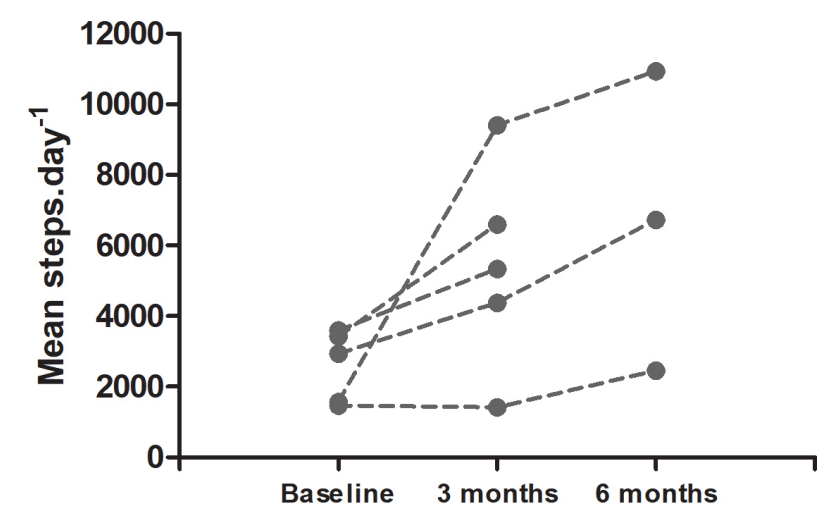

Abstract S21 Figure 1 Daily steps using accelerometry (Actigraph GTX3), at baseline (hospital discharge), 3 months and 6 months for lung transplant recipients assigned to the intervention group $(n=5)$

(by $7 \pm 11$ mins) activities, as well as a reduction in sedentary time (by $-58 \pm 141$ mins) at 3 months. At 6 months $(n=3)$, there was a further improvement in daily steps (by 1641 \pm 661 ), a reduction in sedentary time (by $-28 \pm 137$ mins) and time spent in light activities (by $-21 \pm 36$ mins), with an increase in moderate activity time (by $25 \pm 29$ mins). Following the 3-month intervention, increases were shown in physical functioning, role physical, mental health, bodily pain, and general health SF-36 domain scores, but not in HADs.

Conclusion Tele-coaching appears feasible in lung transplant recipients, with patients wearing the pedometer and interacting well with the app over 3 months. This is promising in the current climate, with the need to develop and evaluate innovative ways of supporting patients remotely.

\section{S22 EVALUATION OF A VIRTUAL PULMONARY REHABILITATION PROGRAMME AND COMPARISON TO TRADITIONAL FACE-TO-FACE PROGRAMMES IN COPD}

${ }^{1} \mathrm{~K}$ Cox, ${ }^{1} \mathrm{~A}$ Krynicka, ${ }^{2} \mathrm{AR}$ Jenkins, ${ }^{3} \mathrm{AW}$ Jones. ' ${ }^{1}$ incolnshire Community Health Services, Lincoln, UK; ${ }^{2}$ Division of Respiratory Medicine, University of Nottingham, Nottingham, UK; ${ }^{3}$ Department of Allergy, Immunology and Respiratory Medicine, Monash University, Melbourne, Australia

\subsection{6/thorax-2021-BTSabstracts.28}

Introduction and Objectives During the coronavirus disease (COVID-19) pandemic, pulmonary rehabilitation (PR) services in the UK ceased traditional face-to-face (F2F) programmes and transitioned to remote or virtual delivery. Virtual programmes may continue to be offered as an alternative to F2F programmes in order to increase accessibility to PR. Our objective was to compare the benefits of Virtual Pulmonary Rehabilitation (VPR) to F2F programmes in a community PR service in England.

Methods With the support of the local Digital Health team, VPR was delivered in cohort groups using Microsoft Teams, twice-weekly for 6 weeks, supported by a specialist physiotherapist and with education delivered live or via pre-recorded webinars. The F2F cohort programme comprised of twiceweekly exercise and education sessions for 6 weeks. Outcomes of VPR delivery (June 2020-March 2021) were compared to F2F programmes from the same time period 12 months previously using the following measures: Chronic Respiratory Questionnaire (CRQ); Hospital Anxiety and Depression Scale (HADS). Due to restrictions, physical capacity for VPR was
Abstract S22 Table 1 Number of patients achieving minimal clinically important differences in CRQ and HADS following Virtual Pulmonary Rehabilitation (VPR) or Face-to-Face pulmonary rehabilitation (VPR)

\begin{tabular}{|c|c|c|c|}
\hline Outcomes & $\begin{array}{l}\text { VPR } \\
\text { n (\%) }\end{array}$ & $\begin{array}{l}\mathrm{F} 2 \mathrm{~F} \\
\mathrm{n}(\%)\end{array}$ & $\begin{array}{l}p \text { value } \\
\text { (Chi-square test) }\end{array}$ \\
\hline CRQ-Dyspnoea & $32(54)$ & $30(55)$ & 0.974 \\
\hline CRQ-Fatigue & $30(50)$ & $34(62)$ & 0.203 \\
\hline CRQ-Emotion & $23(38)$ & $29(53)$ & 0.121 \\
\hline CRQ-Mastery & $24(40)$ & $34(62)$ & 0.019 \\
\hline HADS-Anxiety & $17(33)$ & $24(44)$ & 0.214 \\
\hline HADS-Depression & $12(23)$ & $27(50)$ & 0.004 \\
\hline
\end{tabular}

measured using sit to stand (STS) tests as opposed to Incremental (ISWT) and Endurance Shuttle Walk Tests (ESWT) for F2F.

Results VPR $(n=60)$ produced significantly smaller improvements than F2F $(n=55)$ in CRQ-Fatigue $(+0.4 \pm 1$ vs +0.9 $\pm 1.3, \quad \mathrm{p}=0.036), \quad$ CRQ-Mastery $\quad(+0.3 \pm 1 \quad$ vs. $+0.9 \pm 1.2$, $\mathrm{p}=0.005)$ and HADS-Depression $(-0.1 \pm 2.7$ vs. $-1.9 \pm 3.1$, $\mathrm{p}=0.003)$. Improvements in CRQ-Dyspnoea $(+0.6 \pm 1$ vs. +1 $\pm 1.4, \quad \mathrm{p}=0.159), \quad$ CRQ-Emotion $\quad(+0.3 \pm 1$ vs. $+0.5 \pm 1$, $\mathrm{p}=0.348)$ and HADS-Anxiety $(-0.2 \pm 3$ vs. $-1.4 \pm 3.1, \mathrm{p}=0.132)$ with VPR and F2F were not significantly different. A greater proportion with F2F achieved clinically meaningful improvements in CRQ-Mastery and HADS-Depression but no other statistically significant differences were found (table 1). VPR significantly increased 30 -sec STS $(+1.5$ repetitions, $\mathrm{p}<0.001)$, but not 1 -min STS $(+1.7$ repetitions, $\mathrm{p}=0.085)$. F2F significantly increased ISWT $(+33 \mathrm{~m}, \mathrm{p}<0.001)$ and ESWT $(+180 \mathrm{~m}$, $\mathrm{p}<0.001)$.

Conclusions F2F PR provided larger improvements in outcomes and a greater number of COPD patients achieving meaningful improvements in mastery and depression. However, the number of patients achieving clinically meaningful improvements in dyspnoea, fatigue, emotional function and anxiety with VPR demonstrates the success of transitioning to this model during the pandemic and supports the potential use of such alternative delivery models to increase access to PR.

\section{S23 INTEGRATING HOME-BASED EXERCISE TRAINING WITHIN A HOSPITAL AT HOME SERVICE FOR PATIENTS HOSPITALISED WITH ACUTE EXACERBATIONS OF COPD: A MIXED METHODS FEASIBILITY STUDY}

${ }^{1}$ RE Barker, ${ }^{2} \mathrm{U}$ Brighton, ${ }^{3} \mathrm{~J}$ Bayly, ${ }^{1} \mathrm{JA}$ Walsh, ${ }^{1} \mathrm{CM}$ Nolan, ${ }^{1} \mathrm{~S}$ Patel, ${ }^{1} \mathrm{O}$ Polgar, ${ }^{4} \mathrm{~J}$ Wenneberg, ${ }^{4}$ SSC Kon, ${ }^{5} \mathrm{~A} A$ Wedzicha, ${ }^{2} \mathrm{M}$ Maddocks, ${ }^{6} \mathrm{M}$ Farquhar, ${ }^{1}$ WDC Man. ${ }^{1}$ Royal Brompton and Harefield Hospital, London, UK; ${ }^{2}$ King's College, London, UK; ${ }^{3}$ St Barnabas Hospices, Worthing, UK; ${ }^{4}$ Hillingdon Hospital NHS Foundation Trust, Hillingdon, UK; ${ }^{5}$ Imperial College, London, UK; ${ }^{6}$ University of East Anglia, Norwich, UK

\subsection{6/thorax-2021-BTSabstracts.29}

Background The uptake of face-to-face supervised outpatientbased pulmonary rehabilitation (PR) following hospitalisation for an acute exacerbation of COPD (AECOPD) is low. One commonly cited barrier is travel. Home-based PR may be an alternative setting. The aim of this study was to determine whether a co-designed home-based exercise training intervention, delivered alongside usual hospital at home $(\mathrm{HaH})$ care 
post-hospitalisation for an AECOPD, is acceptable and feasible.

Methods A mixed method feasibility study was conducted including a parallel, two-group randomised controlled trial (RCT) (control group: usual $\mathrm{HaH}$ care; intervention group: usual care plus home-based exercise training) with convergent qualitative components (interviews: patients, family carers, researchers; focus groups: healthcare professionals [HCPs]).

Results 16/132 patients screened were recruited to the RCT with 8 allocated to each group and one withdrawn prior to receiving $\mathrm{HaH}$ care $(56 \%$ were male, mean [SD] age: 74 [9] years, median [IQR] $\mathrm{FEV}_{1}: 29$ [21, 40] percent predicted, $87 \%$ with an eMRC dyspnoea score of $4,5 \mathrm{a}$ or $5 \mathrm{~b}$ ). Four vs eight and four vs seven attended four week and three-month follow-up assessments in the control and intervention groups respectively. There was no evidence of contamination in the control group. $25 \%$ of patients allocated to the intervention group were unable to receive the intervention due to Covid19. The questionnaire-based outcomes were more complete and appeared more acceptable to patients than physical measures, with very poor uptake for physical activity monitoring via accelerometery. Qualitative findings (interviews: five patients, two family carers, four researchers; focus groups: PR and $\mathrm{HaH}$ service HCPs) demonstrated that trial and intervention processes were acceptable, clinically beneficial and safe, but did not explain the disparity between questionnaire-based vs physical outcome measure completion rates.

Conclusion The findings suggest an efficacy trial which investigates home-based exercise training integrated within a $\mathrm{HaH}$ service following hospitalisation for an AECOPD would be safe and acceptable to patients, family carers, HCPs and researchers alike, and is qualitatively felt to be of clinical benefit. However, additional piloting is required to optimise intervention fidelity and study processes given the low recruitment rates, high drop out of the control group and poor uptake of some physical assessments.

\section{S24 IS A NOVEL DIGITAL BREATHING \& ENERGY MANAGEMENT PROGRAMME EFFECTIVE IN REDUCING SYMPTOMS OF LONG COVID?}

J Moore, J Plumbe, N Hilliard, K Plumbe, N Beckett, T Burch, K Bahadur. Respiricare, Kent, UK

\subsection{6/thorax-2021-BTSabstracts.30}

Introduction The most common symptoms of 'Long COVID' which is defined as symptoms $>12$ weeks post COVID infection, are breathlessness and fatigue. Breathing retraining and holistic management for patients suffering with ongoing symptoms of COVID has been recommended to help manage these symptoms. ${ }^{1}$ Ensuring quality rest and activity energy management is essential for the management of chronic fatigue. ${ }^{1,2}$ The aim of this study is to investigate the effectiveness of a novel digital 6-week breathing \& energy group management programme for patients with Long COVID.

Method We conducted a pilot, cohort, observational study using qualitative questionnaires pre and post intervention between Jan -May 2021. The intervention was led by breathing, fatigue specialist physiotherapists and psychological wellbeing practitioners. Baseline information was gathered with an individual digital assessment. Participants were enrolled to weekly digital group sessions focusing on breathing retraining and establishing a good energy management balance. A follow up re-assessment was completed post intervention.

Results 72 participants aged between 24-81, 45 female,27 male, 57 White British, 7 Black British, 2 Black Asian, 6 Other Ethnicity were enrolled. Baseline data showed $87 \%$ $(n=63)$ had a breathing pattern disorder (Breathing Pattern Assessment Tool Score $>$ 4.) 69\% $(n=50)$ had signs of hyperventilation syndrome (Nijmegen score > 23). $77 \%$ were suffering with severe fatigue (Fatigue Severity Scale (FSS) $>5$ ). Outcome measures used were the Self-Reported Chronic Respiratory Disease Questionnaire (SR -CRDQ), General Anxiety Disorder 7 (GAD7), Patient Health Questionnaire PHQ9 and FSS. $86 \%(n=62)$ patients had a clinically significant improvement in at least 1 of the SR-CRDQ domains (breathlessness, emotion, fatigue and mastery).53\% $(\mathrm{n}=38)$ had a clinically significant reduction in FSS. 51\% $(n=37)$ patients had a clinically significant improvement in anxiety or depression.

Conclusion Analysis shows that a digital, novel 6 week breathing and energy management programme was beneficial for patients suffering Long COVID. Continued investigation and further research is required to evaluate the effectiveness of breathing retraining and energy management for patients suffering with Long COVID.

\section{REFERENCES}

1. George PM, et al. Respiratory follow-up of patients with COVID-19 pneumonia. Thorax 2020.

2. Updated NICE guidance on chronic fatigue syndrome. BMJ 2020.

\section{S25 CARDIOPULMONARY EXERCISE TESTING TO EVALUATE EXERCISE LIMITATION AND SHORTNESS OF BREATH IN LONG COVID}

L Godinho, T Wallis, D Levett, S Jack, T Wilkinson, B Marshall, A Banerjee, A Freemans. University of Southampton, Southampton, UK

\subsection{6/thorax-2021-BTSabstracts.31}

Introduction Approximately 10\% of COVID survivors experience long-term sequelae, with fatigue and exercise limitation most frequently reported. The physiological drivers of these symptoms remain unclear. Incremental cardiopulmonary exercise testing (CPET) is a routine clinical assessment used to evaluate exercise limitation but its utility in long COVID is unknown.

Methods Consecutive patients with non-hospitalised SARSCoV2 infection referred for standard-of-care CPET to investigate persistent exercise limitation were identified. Patient demographic and clinical information were extracted, and standard CPET parameters were collected and analysed. Ethical approval was obtained under the UHS REACT COVID observational database (REC-20/HRA/2986).

Results Nine patients were included in this pilot analysis. 55\% male, median (mdn) 47 years, 6 to 12 months post SARSCoV-2 infection. Reported pre-morbid exercise and fitness levels were above average. Patients demonstrated impaired exercise capacity, peak oxygen uptake $\left[\mathrm{VO}_{2}\right.$ peak $]$ (mdn $23.3 \mathrm{ml} / \mathrm{kg} /$ min, $81 \%$ predicted) and oxygen uptake at anaerobic threshold [AT] (mdn $13.4 \mathrm{ml} / \mathrm{kg}$. $/ \mathrm{min}$ ). AT as percentage of $\mathrm{VO}_{2}$ peak was reduced ( $\mathrm{mdn} 45 \%$ ) suggesting significant deconditioning. Oxygen-pulse $\left(\mathrm{O}_{2}\right.$ pulse) percentage predicted was reduced (mdn 80\%) suggesting impaired oxygen delivery and/or muscle oxygen utilisation (table 1). None of the patients demonstrated respiratory limitation to exercise. All patients had normal 